\title{
Preditores do adiamento da aposentadoria por servidores públicos federais
}

\section{Predictores del aplazamiento de la jubilación por servidores públicos federales Predictors of Postponement of Retirement by Federal Public Servants}

\author{
Luciani Soares Silva Macêdo* \\ Pedro F. Bendassolli* \\ Tatiana de Lucena Torres* \\ Universidade Federal do Rio Grande do Norte
}

Doi: http://dx.doi.org/10.12804/revistas.urosario.edu.co/apl/a.4556

Resumo

Este artigo examina a influência de fatores relacionados ao trabalho sobre a intenção de continuar trabalhando além do tempo obrigatório de contribuição. A pesquisa, de corte transversal, foi conduzida com 283 servidores federais brasileiros e envolveu a aplicação da validação brasileira da escala Older Worker's Intention to Continue Working (OWICW) em um survey on-line. Os resultados indicaram que a maioria dos participantes quer continuar no trabalho remunerado. Análises de regressão logística múltipla apontaram como preditores dessa intenção: percepção de autonomia pessoal, interação interpessoal, condições flexíveis de trabalho, além de interesses fora do trabalho. Quanto à decisão de adiar a aposentadoria e permanecer na organização, os fatores preditivos foram: percepção de autonomia pessoal, condições flexíveis de trabalho e o incentivo financeiro (abono de permanência). Os resultados podem subsidiar políticas públicas e de gestão institucional sobre o processo de decisão trabalho-aposentadoria. Palavras-chave: adiamento da aposentadoria, significado do trabalho, servidores públicos, recursos humanos, envelhecimento.

\section{Resumen}

Este artículo analiza la influencia de los factores relacionados con el trabajo en la intención de seguir trabajando más allá del tiempo y de la edad de pensión estatal, en una muestra de 283 servidores públicos brasileños. Se utilizaron cuestionarios en línea con la versión brasileña validada de la escala Intención de los Trabajadores Mayores Seguir Trabajando. Los resultados indicaron que la mayoría quieren seguir en el trabajo remunerado. El análisis de regresión logística

* Universidade Federal do Rio Grande do Norte

Contato principal para correspondência editorial: Luciani Soares Silva Macêdo, Psicóloga e Técnica-administrativa da Universidade Federal do Rio Grande do Norte (UFRN). Doutoranda no Programa de Pós-Graduação em Psicologia da UFRN e membro do Grupo de Estudos e Pesquisa sobre o Trabalho (GEPET/UfRN). Correio eletrônico: lucianissm@gmail.com

Para citar este artigo: Macêdo, L. S. S., Bendassolli, P. F., \& Torres, T. L. (2019). Preditores do adiamento da aposentadoria por servidores públicos federais. Avances en Psicología Latinoamericana, 37(1), 153-167. Doi: http://dx.doi.org/10.12804/ revistas.urosario.edu.co/apl/a.4556 
múltiple mostró como predictores de esta intención: la percepción de la autonomía personal, las relaciones interpersonales, las condiciones de trabajo flexibles e intereses fuera del trabajo. Por otra parte, la percepción de la autonomía personal, condiciones de trabajo flexibles, y los incentivos financieros son predictores de la decisión de posponer la jubilación y permanecer en la organización. El estudio proporciona información que puede apoyar la gestión pública e institucional sobre las políticas de trabajo en el proceso de la jubilación.

Palabras clave: aplazamiento de la jubilación, significado del trabajo, servidores públicos, recursos humanos, envejecimiento.

\section{fbstract}

This article examines the influence of work-related factors on the intention to continue working after state pension age/time. A cross-sectional research was conducted with 283 Brazilians federal employees, and involved an on-line questionnaire with the Brazilian version of the Older Worker's Intention to Continue Working (OwICW) scale. The results indicated that most participants want to continue in paid work. Multiple logistic regression analysis showed that perceptions of personal autonomy at work, interpersonal relationships at work, interests outside of work, and flexible working arrangements are significant predictors of intention to continue working. Furthermore, the perception of personal autonomy at work, flexible working arrangements, the financial incentives are predictors of the decision to postpone retirement and remain in the organization. The study provides information that can contribute with management policies before the process of retirement decision.

Keywords: Postpone retirement, meaning of work, public service, personnel, older workers.

\section{Introdução}

O prolongamento da vida de trabalho tem se caracterizado como uma das principais respostas políticas para o envelhecimento populacional e aumento da longevidade. No âmbito internacional, governos e empregadores estão buscando estratégias para persuadir os trabalhadores a trabalharem mais tempo, podendo-se citar o aumento da idade de aposentadoria, a proibição da aposentadoria compulsória (e.g., USA, Canadá, Austrália), o combate ao ageísmo (estereótipos, preconceitos e discriminação contra os mais velhos), ou mesmo políticas em prol do envelhecimento ativo (Ekerdt, 2010; Phillipson, 2013; Wood, Robertson \& Wintersgill, 2010; Zappalà, Depolo, Fraccaroli, Guglielmi \& Sarchielli, 2008).

Em meio a essas transformações socioeconômicas, a transição trabalho-aposentadoria encontra-se meio imprecisa, podendo avançar o limite estabelecido de idade cronológica ou número de anos trabalhados, a depender de um timing pessoal. Consequentemente, há uma repercussão no meio científico em compreender a decisão de estender a vida de trabalho, caracterizada pelo bridge employment (trabalho após a aposentadoria) ou pela aposentadoria tardia (Flynn, 2010; Menezes \& França, 2012; Zappalà et al., 2008). Este artigo se insere nesta última temática, com o objetivo de examinar fatores relacionados ao trabalho que são preditores da intenção de continuar trabalhando além do tempo obrigatório de contribuição.

Partimos especificamente do prolongamento do trabalho no contexto do serviço público brasileiro. O setor público é um setor competitivo, mas atrativo para os trabalhadores por oferecer diversidade de cargos, estabilidade, boas condições de trabalho, perspectiva salarial e de carreira profissional (Albrecht \& Krawulski, 2011). O emprego do setor público corresponde a cerca de $11 \%$ do total de empregos no Brasil, cujos trabalhadores estão envelhecendo mais rápido (Organização de Cooperação e Desenvolvimento Econômicos [OCDE], 2010). Por exemplo, no âmbito federal, enquanto $56,5 \%$ do total de servidores na ativa já estão acima de 41 anos, cerca de $35 \%$ do total estão acima de 51 anos (Painel Estatístico de 
Pessoal [PEP], 2018). Encaminha-se para a idade mínima de aposentadoria que, atualmente, é de 60 anos para homens e 55 anos para mulheres e requer tempo mínimo de contribuição de 35/30 anos, respectivamente, ressalvadas algumas categorias profissionais (Emenda Constitucional $n^{\circ} 41,2003$ ).

A política de gestão de pessoas sugerida para fazer face ao envelhecimento inclui estratégias para manter os trabalhadores mais velhos além da idade de aposentadoria, principalmente servidores-chave, evitando grandes perdas de memória institucional (OCDE, 2010). A reforma da previdência com aumento da idade mínima e do período de contribuição também é recomendada (OCDE, 2010) e encontra-se em discussão. Medidas como a alteração do limite de idade para a aposentadoria compulsória de 70 para 75 anos (Lei Complementar $\left.\mathrm{n}^{\circ} 152,2015\right)$ já foram implementadas, além do abono de permanência, um benefício equivalente ao valor da contribuição previdenciária, pago ao servidor que tiver preenchido as exigências para aposentadoria voluntária e opte por permanecer em atividade (Emenda Constitucional n ${ }^{\circ} 41,2003$ ). Schettini, Pires \& Santos (2018) apontam que, um quarto dos atuais servidores estão elegíveis à aposentadoria e, dentre as razões pelas quais esse grupo tem optado por continuar trabalhando, o abono de permanência constitui o principal incentivo financeiro.

No Brasil, pesquisas sobre a postergação da aposentadoria são recentes e assinalam as razões financeiras como um dos motivos para o adiamento (Ribeiro, 2012; Pires et al., 2013). No entanto, Menezes \& França (2012) não observaram relação com finanças em um estudo com 148 servidores federais, indicando a partir de análises de regressão logística que idade, controle do trabalho (autoridade de decisão sobre o próprio trabalho), percepção do trabalho (satisfação e envolvimento) e flexibilidade de horário são preditores da decisão de postergar a aposentadoria, sendo os dois últimos também preditores do trabalho após a aposentadoria.

Embora o incentivo financeiro seja apontado entre os melhores preditores da aposentadoria tardia (Tuominen, Karisalmi, Takala \& Kaliva, 2012), o trabalho não se restringe a um meio de sobrevivência. Estudos clássicos revelam que a maioria das pessoas trabalharia mesmo que não houvesse necessidades econômicas, ressaltando que essa atividade preenche outras funções (Harpaz, 2002; Morse \& Weiss, 1955; MOW, 1987). Assim, o que torna o trabalho atrativo de forma que trabalhadores mais velhos optem por permanecer trabalhando? A literatura tem destacado uma variedade de fatores pessoais e relacionados ao trabalho que influenciam essa decisão (Flynn, 2010; França, Menezes, Bendassolli \& Macêdo, 2013), indicando ainda maior preponderância daqueles relacionados ao trabalho (Tuominen et al., 2012).

Vários estudos têm associado razões intrínsecas do trabalho à decisão trabalho-aposentadoria como, por exemplo, o grau de autonomia (Flynn, 2010), flexibilidade (Barnes, Parry \& Taylor, 2004; Pengcharoen \& Shultz, 2010; Shacklock, 2006; Winkelmann-Gleed, 2012), a satisfação com o trabalho (Smeaton \& McKay, 2003), identificação com o trabalho (Barnes et al., 2004), a importância e a centralidade do trabalho (Post, Schneer, Reitman \& Ogilvie, 2013; Tuominen et al., 2012; Zappalà et al., 2008) e o significado do trabalho (Shacklock, 2006; Shacklock \& Brunetto, 2011).

O projeto Meaning of Work (1987) propôs um modelo teórico do significado do trabalho de três dimensões, se tornando uma referência-chave nessa área de pesquisa. As dimensões são: (1) variáveis antecedentes (situação pessoal e familiar, história da carreira e do emprego atual, e ambiente macro socioeconômico), (2) variáveis centrais do significado do trabalho (centralidade do trabalho como um papel da vida, normas sociais sobre o trabalho, resultados valorizados do trabalho, importância das metas do trabalho e identificação 
com o papel laboral) e (3) variáveis consequentes do significado do trabalho (resultados objetivos do trabalho e expectativas subjetivas sobre situações futuras de trabalho) (MOw, 1987).

Segundo a Equipe MOW (1987), os trabalhadores, em suas escolhas relativas ao trabalho, são atraídos para diferentes direções por três tipos de preferências: a remuneração, características intrínsecas do trabalho (e.g., autonomia, variedade, um trabalho interessante e ajustado com as habilidades do indivíduo) e o tempo disponível fora do trabalho. Para optar entre elas, fazem avaliações e compensações em termos de perdas e ganhos, elegendo preferências e escolhas, que por sua vez, estão relacionadas aos seus valores e significado do trabalho.

Shacklock \& Brunetto (2011) utilizaram a estrutura teórica do modelo de significado do trabalho desenvolvido pela equipe MOW (1987), propondo melhor esclarecimento de uma das variáveis consequentes desse modelo: as expectativas subjetivas sobre situações futuras de trabalho. Essa variável diz respeito à importância de trabalhar, às características desejáveis do trabalho e à quantidade de tempo que deve ser dedicada ao mesmo. Desenvolveram para isso a escala Older Worker's Intention to Continue Working (OWICW), validando-a com trabalhadores de uma grande organização do setor público em Queensland, Austrália.

Inspirada nesse estudo, esta pesquisa busca ampliar o diálogo sobre essa temática a partir de dados oriundos do contexto brasileiro. Examina-se que fatores relacionados ao trabalho predizem a intenção de trabalhadores do serviço público federal, em fim de carreira, continuarem trabalhando, preferindo o adiamento da aposentadoria. Estende-se ainda a investigação explorando o papel do recebimento do abono nessa preferência. Intenciona-se, assim, identificar o que os trabalhadores mais velhos buscam no trabalho e na ação do trabalhar, ou seja, suas expectativas e o significado que atribuem ao trabalho.

\section{Método}

\section{Delineamento}

Trata-se de um estudo transversal e descritivo, realizado por meio de um survey, tipo correlacional, utilizando como instrumento de coleta um questionário on-line, auto-administrado. Os pesquisadores disponibilizaram o questionário via e-mail aos servidores que atendiam aos critérios de inclusão: ser servidor ativo permanente, fazendo jus à aposentadoria ou estando até cinco anos antes do tempo previsto. O e-mail foi fornecido por responsável pela gestão de pessoas da instituição. $\mathrm{O}$ acesso ao questionário dependia da anuência do servidor ao termo de compromisso. $\mathrm{O}$ estudo foi aprovado pelo Comitê de Ética, CAAE 08938412.6.0000.5537, seguindo a todos os aspectos éticos e diretrizes dispostas na Resolução CNS 466/12. Ao término, uma devolutiva da pesquisa foi encaminhada aos participantes.

\section{Participantes}

Participaram 283 servidores de uma universidade federal do nordeste brasileiro, do sexo masculino $(n=109)$ e feminino $(n=174)$, dentre os quais $36.4 \%$ eram docentes e $63.6 \%$ técnico-administrativos (TAs). Embora a formação da amostra tenha sido intencional e por conveniência, a relação encontrada entre docentes e TAs representou proporcionalmente bem a distribuição total de servidores da instituição. A idade dos participantes variou de 48 a 69 anos $(M=56.8 ; D P=5.1) \mathrm{e}$ a média do tempo de contribuição foi de 34 anos $(D P=4.4)$. A maior parte informou ser casado ou estar em união estável (65\%) e declarou receber abono de permanência (62.5\%), encontrando-se, pois, em situação de adiamento da aposentadoria. O nível de escolaridade foi elevado, com $71 \%$ já tendo cursado alguma pós-graduação. 


\section{Instrumento}

O questionário on-line possuía duas partes: a escala OwICW, de Shacklock e Brunetto (2011), e uma ficha de dados sócio demográficos contemplando sexo, cargo, situação conjugal, escolaridade, tempo de contribuição à previdência, recebimento de abono, idade planejada para aposentadoria e auto-percepção quanto ao próprio ajustamento na aposentadoria.

A owICW é uma escala numérica constituída por 31 afirmativas, em formato Likert, com gradações de 1 (discordo totalmente) a 7 (concordo totalmente), sendo grande parte delas elaborada a partir do MOW (1987). Os itens se agrupam em sete fatores: vínculo com o trabalho, importância do trabalho, relacionamentos interpessoais, autonomia, condições flexíveis, gestão e fatores organizacionais (aspectos do ambiente e relação supervisor-subordinado) e interesses fora do trabalho. O estudo original ocorreu com 379 funcionários (> 50 anos), apresentando propriedades psicométricas confiáveis $(\alpha>0.70)$ (Shacklock \& Brunetto, 2011). Não foram evidenciadas informações sobre sua aplicação em outras localidades.

A owICW foi disponibilizada pelas autoras, sendo submetida previamente a adaptação transcultural e avaliação da estrutura fatorial para atender aos objetivos deste estudo. Por se tratar da primeira aplicação em contexto brasileiro, foi realizada uma análise fatorial exploratória (AFE) com o método de fatoração dos eixos principais (direct oblimin), suprimindo os itens com carga fatorial inferior a 0.4. A fatorabilidade dos dados disponíveis foi confirmada $(\mathrm{KMO}=0.83$; Teste de esfericidade de Bartlett $=3882.826 ; p<0.001)$.

A versão adaptada apresentou estrutura fatorial relativamente similar para cinco dos sete fatores da estrutura original, havendo como diferencial a subdivisão do fator Interesses Fora do Trabalho em dois, formando um fator separado denominado de influência familiar. A escala com 24 itens (carga fatorial de 0.43 a 0.87 ) ficou agrupada em seis fatores explicando $58.5 \%$ da variância total. $\mathrm{O} \alpha$ de Cronbach total da escala foi de 0.87 .

Os fatores encontrados foram: (1) Autonomia $(\alpha=0.83)$, sobre a percepção de liberdade na efetivação das próprias ideias e decisão de como fazer o seu trabalho; (2) Interação Interpessoal $(\alpha=$ $0.89)$, avaliando benefícios relativos aos contatos sociais no trabalho; (3) Interesses Fora do Trabalho $(\alpha=0.83)$, se há um relacionamento opositor entre outras áreas da vida (desenvolvimento espiritual, interesses comunitários, lazer, hobbies, amigos) e a continuidade do trabalho; (4) Flexibilidade $(\alpha=0.80)$, acerca da possibilidade de trabalhar meio período ou ocasionalmente, poder controlar e escolher as horas de trabalho sem dar satisfação a outros; (5) Influência Familiar ( $\alpha=0.84)$, sobre a influência do cônjuge ou de interesses familiares; e por fim, (6) Vínculo com o Trabalho $(\alpha=0.69)$, investigando sobre a importância e a identificação do sujeito com o trabalho.

Variável critério. A principal variável critério, a intenção de continuar trabalhando, foi medida pela afirmativa "eu quero continuar no trabalho remunerado". Para melhor elucidar essa intenção, duas afirmativas foram acrescentadas na versão brasileira: "eu vou querer adiar a aposentadoria e permanecer no emprego atual", e "eu vou querer me aposentar do emprego atual e trabalhar em outro lugar".

Adicionalmente, também foi mensurada a intenção de aposentar-se utilizando quatro afirmativas que relacionavam a intenção de parar o trabalho remunerado ao desejo de: passar mais tempo com o cônjuge e com a família, ter menos pressão e prazos a cumprir, e ter mais escolhas e maior flexibilidade. Neste estudo, o $\alpha$ de Cronbach destes itens foi de 0.79 . Com os escores estimados pela média de pontos atribuídos a essas questões foi originada uma quarta variável-resultado: “eu vou querer parar o trabalho remunerado". 


\section{Procedimentos de análise dos dados}

Dos 320 questionários obtidos foram considerados válidos apenas os sem missings, ou seja 283, sendo analisados com o auxílio do Statistical Package for the Social Sciences (SPSS). A ocorrência de problemas de normalidade na distribuição dos dados, verificada pelo teste de Kolmogorov-Smirnov $(p<0.05)$, conduziu à escolha de análises não paramétricas.

Para examinar os preditores da intenção de continuar trabalhando foram construídos modelos de regressão logística múltipla entre os fatores e as variáveis de saída. A regressão logística é uma técnica mais flexível por não considerar a hipótese de normalidade e linearidade entre as variáveis. Seus resultados são interpretados a partir do valor Exp $b$ das variáveis preditoras, que indica que quando o previsor aumenta uma unidade, as chances de a variável de saída ocorrer também aumentam (Exp $b>1)$ ou diminuem $(\operatorname{Exp} b<1)$. Apresenta ainda, a estatística Nagelkerke $\mathrm{R}^{2}$ que é semelhante ao $\mathrm{R}^{2}$ do modelo de regressão múltipla linear. $\mathrm{O}$ método de regressão empregado foi o Stepwise Backward Likelihood Ratio (Tabachnick \& Fidell, 2006; Field, 2009).

Os escores dos participantes para cada fator foram estimados com base na média dos pontos atribuídos aos itens que o constituíam. Como as variáveis de saída eram afirmativas em formato Likert de sete pontos, cada uma delas foi transformada em uma variável binária (discordo vs. concordo) na planilha de dados. Os participantes que declararam algum grau de concordância (valores de 5 a 7) tiveram o escore computado como " 1 ", e os que não declararam essa opinião (valores de 1 a 4) foram considerados como " 0 ".

Os dados sócios demográficos foram utilizados para caracterizar a amostra e segmentá-la, explorando diferenças entre grupos (teste $U$, de Mann-Whitney). O tamanho do efeito relatado se baseia na classificação de Cohen (1988, 1992, citado por Field, 2009) onde: $r=0.10$ (efeito pe- queno), $r=0.30$ (efeito médio), e $r=0.50$ (efeito grande). Para todas as análises realizadas, considerou-se como nível de significância $p<0.05$.

\section{Resultados}

Nesta seção, apresenta-se os resultados concernentes ao tópico desse estudo: a identificação de fatores relacionados ao trabalho que são atrativos e explicam a intenção de continuar trabalhando além do tempo obrigatório de contribuição. Essa intenção foi investigada em duas direções: do adiamento da aposentadoria e permanência no emprego atual, ou do trabalho após a aposentadoria. Complementarmente, explora-se a relação entre esses fatores e a intenção de aposentar-se. Por último, situa-se os resultados descritivos e de diferença entre médias das intenções, relacionados aos dados sócios demográficos dos participantes.

\section{Intenção de continuar trabalhando}

Para examinar que fatores relacionados ao trabalho poderiam melhor explicar a intenção de continuar trabalhando, foi conduzida uma análise de regressão logística com a variável critério "eu quero continuar no trabalho remunerado". Os resultados dessa análise são apresentados na tabela 1 .

Explorando os relacionamentos entre as variáveis, somente quatro fatores (autonomia, interação interpessoal, interesses fora do trabalho e vínculo com o trabalho) apresentaram escores significativos $(p<0.01)$ com potencialidade de contribuição para o modelo. Porém, verificou-se a inclusão do fator Flexibilidade como preditor no modelo construído sem que o mesmo fosse correlacionado diretamente com a intenção de continuar trabalhando $(p=0.72)$.

Detectando que a remoção desse fator afetaria negativamente a habilidade preditiva do modelo ( $p=0.004)$, e descartada sua permanência como resultado de multicolinearidade entre as variáveis previsoras, foi observado que seu surgimento ocorria 
apenas em interação com o fator Autonomia, aumentando significativamente $(p=0.019)$ a variância de explicação de $12 \%$ para $15 \%$ (Nagelkerke $\mathrm{R}^{2}$ ). Assim sendo, identificou-se que o fator Flexibilidade atuava com um efeito moderador no modelo de predição em interação com o fator Autonomia.

$\mathrm{Na}$ análise executada, associando o fator Flexibilidade aos quatro fatores que apresentaram escores significativos, dois modelos de predição foram construídos. O Modelo 2, com a estatística da razão de verossimilhança apresentando $\chi^{2}(4)=44.21$; $p<0.001$, foi o que melhor identificou os fatores relacionados à intenção de continuar trabalhando explicando $20.3 \%$ (Nagelkerke $\mathrm{R}^{2}$ ) da variância. O modelo encontrado classificou corretamente $72 \%$ dos participantes (destes, $89.6 \%$ que concordaram e $34.4 \%$ dos que discordaram), explicando melhor os que manifestaram a intenção de continuidade. O teste de Hosmer \& Lemeshow não significativo $(p>0.05)$ indicou bom ajuste do modelo.

Tabela 1.

Regressão logística múltipla: preditores da intenção de continuar trabalhando

\begin{tabular}{llccc}
\hline \multicolumn{1}{c}{ Preditores } & $\beta$ & EP & $\operatorname{Exp} b[\mathrm{IC} 95 \%]$ \\
\hline & Autonomia & $0.42^{* * *}$ & 0.11 & $1.52[1.23 ; 1.89]$ \\
& $\begin{array}{l}\text { Interação in- } \\
\text { terpessoal }\end{array}$ & 0.22 & 0.12 & $1.25[0.98 ; 1.60]$ \\
Passo 1 & $\begin{array}{l}\text { Interesses fo- } \\
\text { ra do trabalho }\end{array}$ & $0.26^{*}$ & 0.11 & $1.30[1.06 ; 1.60]$ \\
& $\begin{array}{l}\text { Flexibilidade } \\
\text { Vínculo }\end{array}$ & $-0.28^{* *}$ & 0.10 & $0.75[0.62 ; 0.92]$ \\
& 0.09 & 0.14 & $1.10[0.84 ; 1.43]$ \\
Constante & -2.52 & 0.73 & \\
\hline & $\begin{array}{l}\text { Autonomia } \\
\text { Interação in- } \\
\text { terpessoal }\end{array}$ & $0.42^{* * *}$ & 0.11 & $1.52[1.23 ; 1.88]$ \\
Passo 2 & $\begin{array}{l}\text { Interesses fo- } \\
\text { ra do trabalho }\end{array}$ & $0.26^{*}$ & 0.11 & $1.30[1.04 ; 1.62]$ \\
& Flexibilidade & $-0.28^{* *}$ & 0.10 & $0.75[0.62 ; 0.92]$ \\
Constante & -2.25 & 0.62 & \\
\hline
\end{tabular}

Nota. $\mathrm{R}^{2} \%=20.3 \%\left(\right.$ Nagelkerke $\left.\mathrm{R}^{2}\right)$

$* \mathrm{p}<0.05 ; * * \mathrm{p}<0.01 ; * * * \mathrm{p} \leq 0.001$
Em síntese, os resultados do Modelo 2 (tabela 1) indicaram que uma maior percepção de autonomia no trabalho $(p<0.001)$, de benefícios da interação interpessoal ( $p=0.022)$, e interesses fora do trabalho $(p=0.013)$, tornam os indivíduos de 30 a $52 \%$ mais propensos a decidir por continuar no trabalho remunerado, dado certo nível de flexibilidade $(p=0.006)$. $\mathrm{O}$ fator Vínculo com o Trabalho não se mostrou um previsor significativo no contexto da análise multivariada, mas isoladamente torna $34 \%$ mais provável a intenção de continuar no trabalho remunerado $\operatorname{com} \beta=0.296(E P=0.112)$, $p=0.008 ; \operatorname{Exp} b=1.34 ;$ IC 95\% [1.08; 1.68].

Adiar a aposentadoria elou trabalhar após a aposentadoria. Para esclarecer sobre os fatores preditivos da intenção do adiamento, foi conduzida uma análise de regressão logística com a variável critério "eu vou querer adiar a aposentadoria e permanecer no emprego atual". Os resultados dessa análise são apresentados na tabela 2 .

Inicialmente, apenas dois fatores apresentaram escores com potencialidade de contribuição para o modelo: autonomia $(p<0.05)$ e interação interpessoal $(p<0.01)$. Diante da potencialidade do conjunto de variáveis, atestado pelo valor da estatística global $(p<0.01)$, utilizou-se como critério de inclusão nesta análise as variáveis cujo valor descritivo de significância do teste fosse menor que 0.20 (Hosmer \& Lemeshow, 2000, citado por Tabachnick \& Fidell, 2006). Com esse procedimento, mais dois fatores foram incluídos (flexibilidade e vínculo com o trabalho) mantendo a significância de $p$ da estatística global.

Com a combinação desses quatro fatores, dois modelos de predição foram construídos. O Modelo 2 , com a estatística da razão de verossimilhança $\chi^{2}$ (3) $=16.41 ; p=0.001$, foi o que melhor identificou os previsores da intenção de adiar a aposentadoria e permanecer no emprego atual, explicando $7.6 \%$ (Nagelkerke $\mathrm{R}^{2}$ ) da variância dessa intenção. 
Tabela 2.

Regressão logística múltipla: preditores da intenção de adiar a aposentadoria

\begin{tabular}{llccc}
\hline \multicolumn{1}{c}{ Preditores } & $\beta$ & EP & Exp $b[\mathrm{IC} 95 \%]$ \\
\hline $\begin{array}{l}\text { Autonomia } \\
\text { Interação in- } \\
\text { terpessoal }\end{array}$ & $0.21^{*}$ & 0.10 & $1.24[1.02 ; 1.50]$ \\
Passo 1 & Flexibilidade & $-0.24 * *$ & 0.09 & $0.78[0.66 ; 0.93]$ \\
& Vínculo & 0.01 & 0.12 & $1.01[0.80 ; 1.30]$ \\
& Constante & -0.64 & 0.63 & \\
\hline & Autonomia & $0.21 *$ & 0.10 & $1.24[1.02 ; 1.50]$ \\
Passo 2 & $\begin{array}{l}\text { Interação in- } \\
\text { terpessoal }\end{array}$ & 0.20 & 0.10 & $1.22[1.00 ; 1.49]$ \\
& Flexibilidade & $-0.24 * *$ & 0.09 & $0.78[0.66 ; 0.93]$ \\
& Constante & -0.60 & 0.53 &
\end{tabular}

Nota. $\mathrm{R}^{2} \%=7.6 \%$ (Nagelkerke $\left.\mathrm{R}^{2}\right)$

$* \mathrm{p}<0.05 ; * * \mathrm{p}<0.01 ; * * * \mathrm{p} \leq 0.001$

O Modelo 2 obteve bom ajuste (Hosmer \& Lemeshow $\operatorname{com} p>0.05$ ) não apresentando nenhum outlier, e classificou corretamente $64.7 \%$ da amostra. Destes, previu 89,1\% com intenção de adiamento e $25.7 \%$ dos que não manifestaram essa intenção. Os resultados indicaram que a combinação das variáveis autonomia $(p=0.033)$ e flexibilidade $(p=0.006)$ (tabela 2$)$ torna o indivíduo $24 \%$ mais propenso a adiar a aposentadoria e permanecer na organização, a depender do nível de flexibilidade percebida. A aspiração por maior flexibilidade (média $>5$ ) reduz as chances de ocorrência do adiamento em $22 \%$.

Embora o fator Interação Interpessoal no Trabalho tenha isoladamente sido apresentado como preditor $\operatorname{com} \beta=0.248$ ( $E P=0.092), p=0.007$; $\operatorname{Exp} b=1.28$; IC 95\% [1.07; 1.54], não alcançou significância estatística na análise multivariada $(p=0.52)$. O fator Vínculo com o Trabalho não contribuiu para este modelo.

Expandindo essa investigação a fim de explorar o impacto do incentivo financeiro (abono de permanência) para a decisão do adiamento, reali- zou-se uma nova análise de regressão, adicionando uma variável categórica sobre o recebimento do abono $(0=$ não; $1=$ sim $)$. Em comparação com a análise anterior, o novo modelo, que apresentou $\chi^{2}(4)=37.87 ; p<0.001$, aumentou o poder de explicação da variância da decisão de adiamento de $7.6 \%$ para $17 \%$ (Nagelkerke $\mathrm{R}^{2}$ ). Classificou corretamente $66.4 \%$ dos participantes, sendo $81 \%$ dos que tinham a intenção de adiamento, aumentando a previsão dos que não manifestaram essa intenção para $43.1 \%$. Tendo igualmente apontado a autonomia e flexibilidade como preditores, essa análise acrescentou que o recebimento do abono de permanência, com $\beta=1.22(E P=0.27)$, $p<0.001 ; \operatorname{Exp} b=3.39$; IC 95\% [2.00; 5.74], torna os participantes 3.39 vezes mais prováveis de adiar a aposentadoria, enquanto que o não recebimento reduz aproximadamente em $70 \%$ as chances de adiamento.

Para esclarecer sobre os fatores preditivos da intenção de trabalhar após a aposentadoria, foi utilizada a variável critério "eu vou querer me aposentar do emprego atual e trabalhar em outro lugar". Embora cinco fatores (autonomia, interação interpessoal, interesses fora do trabalho, flexibilidade e influência familiar) tenham apresentado escores com significância menor que 0.20 (Hosmer \& Lemeshow, 2000, citado por Tabachnick \& Fidell, 2006), a estatística qui-quadrado dos resíduos $(p>0.05)$ informou a impossibilidade de um modelo significativo de previsão. Selecionando apenas três fatores (interesses fora do trabalho, influência familiar e autonomia), que apresentaram valor de $p$ de 0.01 a 0.07 , se tornou possível a construção de um modelo ( $p=0.038)$.

Entretanto, como a análise considera como referência o evento com maior número de participantes, em vez de se obter os preditores da intenção de trabalhar após a aposentadoria $(n=94)$, obteve-se os preditores da intenção de não trabalhar após a aposentadoria $(n=189)$. Os resultados das análises foram semelhantes ao utilizar o método 
Backward ou Forward, optando-se por relatar esse último pela simplicidade da descrição.

Apenas um único modelo foi construído com bom ajuste (Hosmer \& Lemeshow $\operatorname{com} p>0.05$ ) e sem outliers, apresentando estatística da razão de verossimilhança $\chi^{2}(1)=6.88 ; p<0.01$. O fator Interesses Fora do Trabalho, isoladamente, explica $3.3 \%$ (Nagelkerke $\mathrm{R}^{2}$ ) da variância dessa intenção $\operatorname{com} \beta=0.223(E P=0.086), p=0.01 ; \operatorname{Exp} b=$ 1.25 ; IC 95\% [1.06; 1.48]. Esse dado indica que, a cada aumento desse previsor em uma unidade, a chance de não trabalhar após a aposentadoria aumenta em $25 \%$.

\section{Aposentar-se e parar o trabalho remunerado}

Complementarmente, a existência de previsores da intenção de parar o trabalho remunerado foi explorada realizando-se uma regressão logística com uma quarta variável critério "eu vou querer parar o trabalho remunerado para...". Tal medida explora razões que justificariam a intenção de aposentar-se relacionada ao desejo de ter mais tempo para os relacionamentos familiares, ter mais escolhas, maior flexibilidade e liberdade do trabalho (pressão e prazos), permitindo verificar a congruência de outras respostas dos participantes. Os resultados dessa análise são apresentados na tabela 3.

Todos os seis fatores foram incluídos no modelo de investigação por apresentarem nível de significância entre 0.001 e 0.076 e estatística global com $p<0.001$ (Hosmer \& Lemeshow, 2000, citado por Tabachnick \& Fidell, 2006). Durante a análise, foram construídos dois modelos de predição (tabela 3), sendo o Modelo 2, com a estatística da razão de verossimilhança apresentando $\chi^{2}(5)=48.34$; $p<0.001$, o que melhor identificou os previsores influentes da decisão de parar o trabalho remunerado, explicando $20.9 \%$ (Nagelkerke R Square) da variação dessa intenção.

O Modelo 2 classificou corretamente $66.4 \%$ do total de predições, sendo destas $72.1 \%$ dos que declararam intenção de parar o trabalho re-
Tabela 3.

Regressão logística múltipla: preditores da intenção de aposentar-se

\begin{tabular}{|c|c|c|c|c|}
\hline & Preditores & $\beta$ & EP & $\operatorname{Exp} b[$ IC 95\%] \\
\hline \multirow{7}{*}{ Passo 1} & Autonomia & $-0.37 * * *$ & 0.11 & $0.69[0.56 ; 0.86]$ \\
\hline & $\begin{array}{l}\text { Interação in- } \\
\text { terpessoal }\end{array}$ & -0.17 & 0.12 & $0.84[0.66 ; 1.06]$ \\
\hline & $\begin{array}{l}\text { Interesses } \\
\text { fora do tra- } \\
\text { balho }\end{array}$ & 0.18 & 0.10 & $1.20[0.98 ; 1.47]$ \\
\hline & Flexibilidade & $0.31 * * *$ & 0.10 & $1.36[1.13 ; 1.64]$ \\
\hline & $\begin{array}{l}\text { Influência } \\
\text { familiar }\end{array}$ & $0.24 * *$ & 0.08 & $1.28[1.09 ; 1.50]$ \\
\hline & Vínculo & -0.09 & 0.13 & $0.91[0.71 ; 1.18]$ \\
\hline & Constante & 0.64 & 0.67 & \\
\hline \multirow{6}{*}{ Passo 2} & Autonomia & $-0.37 * * *$ & 0.11 & $0.69[0.56 ; 0.86]$ \\
\hline & $\begin{array}{l}\text { Interação in- } \\
\text { terpessoal }\end{array}$ & $-0.21 *$ & 0.11 & $0.81[0.66 ; 1.00]$ \\
\hline & $\begin{array}{l}\text { Interesses } \\
\text { fora do tra- } \\
\text { balho }\end{array}$ & 0.18 & 0.10 & $1.19[0.98 ; 1.46]$ \\
\hline & Flexibilidade & $0.31 * * *$ & 0.10 & $1.36[1.13 ; 1.64]$ \\
\hline & $\begin{array}{l}\text { Influência } \\
\text { familiar }\end{array}$ & $0.25 * *$ & 0.08 & $1.28[1.09 ; 1.50]$ \\
\hline & Constante & 0.39 & 0.56 & \\
\hline
\end{tabular}

Nota. $\mathrm{R}^{2} \%=20.3 \%\left(\right.$ Nagelkerke $\left.\mathrm{R}^{2}\right)$

$* \mathrm{p}<0.05 ; *$ p $<0.01 ; * * * \mathrm{p} \leq 0.001$

munerado e $60.3 \%$ dos que não declararam essa intenção. Apresentando um bom ajuste (Hosmer $\&$ Lemeshow com $p>0.05)$, os resultados mostraram que a influência familiar $(p=0.002)$ torna $28 \%$ mais provável a intenção de parar o trabalho remunerado. Semelhantemente, a flexibilidade $(p=0.001)$, intensificada pela aposentadoria, também aumenta a chance dessa intenção em 36\%. Coerentemente com o modelo construído sobre a intenção de continuar trabalhando, os benefícios dos relacionamentos interpessoais no trabalho e uma maior percepção de autonomia reduzem a intenção de aposentar-se em 19\% e 31\%, respectivamente. 
O fator interesses fora do trabalho apresentou um potencial de relacionamento positivo para essa decisão, mas não alcançou a significância estatística $(p=0.85)$ na análise multivariada. Os intervalos de confiança desse fator cruzam a unidade (1) indicando certa instabilidade da direção desse relacionamento na população, podendo tanto ser negativa $(\operatorname{Exp} b<1)$ quanto positiva $(\operatorname{Exp} b>1)$.

\section{Variáveis sócio demográficas e a intenção de continuar trabalhando}

Do ponto de vista descritivo, a maioria dos participantes desta pesquisa $(68.2 \%)$ declararam intenção de continuar no trabalho remunerado além do tempo obrigatório de contribuição. Porém, esta intenção tende mais ao adiamento da aposentadoria e permanência na própria organização $(61.5 \%)$ do que a trabalhar em outro lugar após a aposentadoria (33.2\%).

Análises bivariadas com o teste $U$ de Mann-Whitney revelaram diferenças significativas quanto a intenção de continuar ou parar de trabalhar. Indivíduos que discordaram quanto a um fácil ajustamento na aposentadoria apresentaram maior intenção de continuar trabalhando ( $U=6847.50 ; r=-0.21)$ e maior intenção de adiar a aposentadoria $(U=6033.50 ; r=-0.28)$ do que os que acreditavam em um fácil ajustamento. Esses efeitos foram relatados como significativos a $p \leq 0.001$ (bicaudal).

Por sua vez, os participantes que concordaram com um fácil ajustamento na aposentadoria apresentaram maior intenção de parar o trabalho remunerado do que aqueles que discordaram ( $U=7075$; $r=-0.18)$. Semelhantemente, os participantes casados ou que se encontravam em união estável apresentaram maior intenção de parar o trabalho remunerado, diferindo dos que não possuíam parceiros $(U=7086 ; r=-0.18)$. Esses efeitos foram relatados como significativos a $p<0.01$ (bicaudal).
Já os servidores que recebem o abono de permanência têm maior intenção de adiar a aposentadoria do que os que não recebem $(U=6941$; $p<0.001$ bicaudal; $r=-0.22$ ). Com relação ao sexo, os dados apontam que os servidores homens apresentaram maior intenção de trabalhar após a aposentadoria, diferindo estatisticamente das mulheres $(U=7615.50 ; p<0.01$ bicaudal; $\mathrm{r}=-0.17)$.

Também foi observado que os docentes $(M d=65)$ planejam se aposentar com mais idade do que os TA $(M d=60)(U=5397 ; \mathrm{r}=-0.34)$ e planejam parar de trabalhar em qualquer trabalho remunerado com mais idade $(M d=70)$ do que os TA $(M d=65)(\mathrm{U}=5332.50 ; \mathrm{r}=-0.32)$, sendo esses efeitos relatados ao nível $p<0.001$ (bicaudal).

\section{Discussão}

Os resultados revelam que a maioria dos participantes tem a intenção de continuar no trabalho remunerado além do período de contribuição obrigatória. Mas, para a população estudada, a tendência de prolongamento da vida laboral se refere mais ao adiamento da aposentadoria e permanência na instituição atual do que ao trabalho após a aposentadoria. Como a maioria dos participantes já se encontram em situação de adiamento, trata-se de uma decisão concretizada e não apenas de uma intenção. Para facilitar a compreensão do leitor, os modelos construídos pela regressão logística foram sintetizados em uma figura identificando os preditores encontrados.

A constatação do abono de permanência como um preditor importante vem reforçar a influência financeira sobre as escolhas relativas ao trabalho, já apontada na literatura (e.g., Barnes et al., 2004; Flynn, 2010). MOW (1987) igualmente destaca o "papel da remuneração como um reforço condicionado generalizado" (p. 156). Percebemos assim, correspondentemente ao encontrado por Tuominen et al. (2012), que o incentivo financeiro tem se mostrado uma política eficaz à aposentadoria tardia. 
Fatores

Variáveis de saida

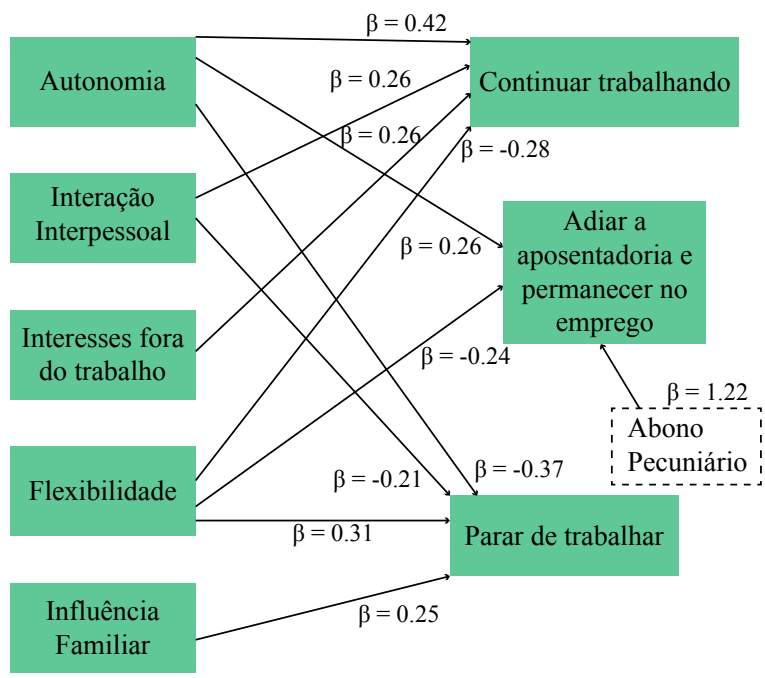

Figura 1. Fatores preditivos da intenção de continuar ou parar de trabalhar

Mesmo assim, características desejáveis do trabalho devem ser possibilitadas. Para os trabalhadores mais velhos, os valores que tornam o trabalho atrativo, interessante e gratificante nessa etapa de vida, fazendo parte de suas expectativas subjetivas, são um trabalho que permita a autonomia pessoal, oportunize a interação interpessoal, ofereça uma flexibilidade razoável e esteja atrelado a outros interesses fora do trabalho. Ao apontar essas características intrínsecas como desejadas, eles evidenciam que consideram o trabalho uma das vias de acesso à satisfação dessas necessidades.

Os dois fatores iniciais, a autonomia e a interação interpessoal, aparecem aumentando significativamente a chance de continuar trabalhando, $\mathrm{e}$ congruentemente, reduzindo as chances de parar o trabalho remunerado. A autonomia se refere à liberdade de tomar decisões relativas ao exercício das funções e na realização de tarefas. Particularmente, foi o aspecto atrativo que mostrou maior associação, sendo consistente como preditor da intenção de continuar no trabalho remunerado e da decisão de adiamento e permanência no vínculo atual. A importância desse fator já foi observada por outros autores (Harpaz, 2002; Morin, 2001; Shacklock, 2006), sendo até considerada um dos valores universais do trabalho diante da proporção de pessoas que preferem essa característica (MOW, 1987).

O segundo fator corrobora que relacionamentos interpessoais positivos no trabalho tornam mais provável a intenção de continuar trabalhando. A oportunidade de manter contato com pessoas no trabalho é apontada como uma das perdas na aposentadoria e uma das razões de continuidade laboral (Barnes et al., 2004; França \& Vaughan, 2008; Morin, 2001; Morse \& Weiss, 1955; MOW, 1987; Pires et al., 2013; Winkelmann-Gleed, 2012), enquanto o baixo nível de relações interpessoais no trabalho prediz a descontinuidade (Harpaz, 2002).

A importância desse fator pode estar relacionada ao fato que, para alguns, a necessidade de contatos e relacionamentos interpessoais são mais preenchidas no ambiente de trabalho do que no ambiente externo, como sugerido por Harpaz (2002). Outra consideração a esse respeito é levantada no estudo de Morin (2001), que indica as experiências satisfatórias de interação humana no trabalho como uma das características de um trabalho que tem sentido, contribuindo não somente para o desenvolvimento da identidade pessoal e social, como também para a transposição de questões existenciais referentes à solidão e morte.

O terceiro fator que contribuiu com o modelo é o da flexibilidade (trabalhar meio período, poder escolher e controlar as horas de trabalho). Embora seja apontada na literatura como um aspecto desejável (Barnes et al., 2004; Menezes \& França, 2012; Pengcharoen \& Shultz, 2010; Shacklock, 2006; Shacklock \& Brunetto, 2011; Winkelmann-Gleed, 2012), conforme os achados, a flexibilidade isoladamente não prediz a intenção de continuar trabalhando. No entanto, no contexto da análise multivariada, esse fator se mostrou uma variável moderadora associada à autonomia, 
podendo aumentar ou diminuir a relação desta com a intenção de continuar, a depender do nível de flexibilidade percebida.

Uma possível explicação para isso é que, até certo ponto, condições flexíveis quanto aos horários de trabalho e na escolha de quando completar suas tarefas contribui para a percepção de liberdade e o sentimento de responsabilidade no exercício da atividade, aumentando o grau de autonomia percebida. No entanto, o desejo por uma maior flexibilidade, ao ponto de trabalhar ocasionalmente e não precisar dar satisfações a outros, por ser difícil de ser concretizada no âmbito da instituição, atuaria como redutor da intenção de continuar e potencializador das chances de aposentar-se.

$\mathrm{O}$ quarto fator se refere aos interesses fora do trabalho (hobbies, amigos, desenvolvimento espiritual, lazer e os interesses comunitários). Semelhantemente ao estudo de Shacklock \& Brunetto (2011), esse fator se revelou como um preditor da intenção de continuar trabalhando, embora outros estudos indiquem uma relação positiva desses interesses com a aposentadoria (França, 2009; França \& Vaughan, 2008; Phillipson \& Smith, 2005). Esse dado sugere uma fronteira mais frágil entre o trabalho e o não-trabalho, sinalizando que, quando o lugar ocupado pelo trabalho intermedia outros espaços da vida pessoal, este se torna mais atrativo.

Mas, os resultados deste estudo diferem em parte do encontrado por Shacklock \& Brunetto (2011). $\mathrm{Na}$ amostra australiana, os preditores da intenção de continuar no trabalho remunerado encontrados são: condições flexíveis de trabalho, interesses fora do trabalho, gestão e fatores organizacionais e importância do trabalho para o indivíduo. Porém, na adaptação brasileira, esses dois últimos fatores não apresentaram bom desempenho.

No que diz respeito à intenção de aposentar-se do emprego atual e trabalhar em outro lugar, não foi possível investigar os fatores preditivos nesta direção. Diante da estabilidade que caracteriza o serviço público, a opção do adiamento pareceu ser mais atrativa do que o trabalho após a aposentadoria, o que pode ser devido aos custos emocionais envolvidos com a quebra de vínculo pela aposentadoria e a insegurança em construir novas alternativas de carreira. Os resultados apontam apenas que os servidores homens têm maior intenção de trabalhar após a aposentadoria do que as mulheres, condizente com o encontrado por Tuominen et al. (2012).

Por fim, com relação à intenção de se aposentar, surgiram a combinação de quatro fatores preditivos e concorrentes. Como o papel preditivo da autonomia e interação interpessoal reduzindo a chance de aposentar-se é coerente com os resultados já discutidos inicialmente, restringe-se agora a discutir o papel dos outros dois fatores.

A influência familiar, representada pelo desejo de passar mais tempo com familiares e cônjuge, surge em oposição aos relacionamentos proporcionados pelo trabalho. Seu impacto, aumentando as chances de aposentar-se, encontra suporte na literatura (França, 2009; Smeaton \& McKay, 2003), particularmente para os que possuem vínculos familiares fortes (Szinovacz, deViney \& Davey, 2001), condizendo com os que têm situação conjugal estável.

O papel do fator Flexibilidade neste modelo não fica de todo claro, pois aqui ele possui um papel preditivo próprio e não como moderador. Por um lado, a percepção de autonomia no trabalho, tendo liberdade de decisão em sua atividade, reduz a chance de aposentar-se. Por outro, a flexibilidade intensificada pela aposentadoria, de ter menos pressão e prazos a cumprir, de poder escolher quando e como se dedicar às atividades (e dentre elas o trabalho, se assim o desejar), tornam a aposentadoria mais provável. Uma possível explicação para isto é que a atração por condições mais flexíveis e a impossibilidade de sua satisfação no âmbito da instituição, somado a um contexto de pressão e intensificação do trabalho, remeta à liberdade proposta pela aposentadoria aumentando as chances dessa decisão, como também observado por Winkelmann-Gleed (2012). 
Diante do exposto, é importante sinalizar que este estudo apresenta algumas limitações, o que fomenta possibilidade de pesquisas futuras. Primeiramente, limita-se quanto à sua generalização por incidir apenas sobre servidores de uma universidade, com seus resultados se aplicando a uma população específica. Estudos com outros tipos de trabalhadores mais velhos poderiam esclarecer melhor sobre as expectativas subjetivas a respeito do trabalho nessa fase da vida.

Em segundo lugar, por se tratar de uma pesquisa de levantamento descritiva e transversal, verificando opiniões em um determinado espaço de tempo, pode estar expressando uma visão que não será, obrigatoriamente, a realidade prática acerca das preferências de trabalho e aposentadoria daqui a alguns anos. Outros fatores, além do trabalho, influenciam as decisões. Um exemplo disso foi o aumento expressivo de pedidos de aposentadoria de servidores federais no ano de 2017 (42.40\%), quando comparado ao ano de 2016 (PEP, 2018). Esse fato parece ser um efeito das discussões sobre a reforma da previdência na época e ameaças de extinção do abono de permanência como medida para redução de gastos públicos (e.g., a PEC 139/15). Conforme Bôas \& Souza (2015), analistas apontam que o fim do abono provocaria um comportamento massivo de aposentadoria. Um estudo de seguimento poderia trazer mais esclarecimentos a esse respeito.

Em terceiro, observamos que os fatores preditivos encontrados, relacionados ao trabalho, alcançaram baixo poder explicativo da variância da intenção de continuar trabalhando (17 a 20\%) e explicam melhor os que concordam com a continuidade. Tal fato indica que outras variáveis não contempladas poderiam ter um papel igualmente influente ou complementar, respondendo pelos $80 \%$ restantes da variância. Por exemplo, a idade em que se planeja continuar no trabalho remunerado pode ser uma influência. A constatação de que os docentes planejam permanecer mais tempo no mercado de trabalho do que os TAs, também sugere haver alguma relação entre a atividade da docência em si e a continuidade do trabalho. Teríamos os mesmos preditores com trabalhadores de instituições/organizações privadas, com outras atividades que incluem desde tarefas extremamente repetitivas, até outras que exigem intenso desgaste físico? Essas são questões que, a nosso ver, podem enriquecer, ao ampliar, as dimensões que constituem o significado do trabalho e suas relações com a intenção de trabalhar ou aposentar-se. Novos estudos poderiam explorar a influência do tipo de atividade e seu contexto sobre variáveis preditoras da continuidade do trabalho, notadamente, variáveis relativas ao sentido do trabalho.

Diante do crescente interesse mundial de investigar maneiras eficientes de incentivar e aumentar a participação de trabalhadores mais velhos no mercado de trabalho, implicações éticas e práticas precisam ser consideradas. $\mathrm{O}$ estímulo ao adiamento da aposentadoria exige políticas de gestão de pessoas voltadas para esta continuidade laboral, com atenção especial ao ageísmo e a maior possibilidade de doenças ocupacionais e crônicas com o envelhecimento.

Outra questão é que, a relação encontrada entre a intenção de continuar trabalhando e a percepção de um difícil ajustamento à aposentadoria reforça o desafio e a importância da atuação dos programas de preparação para a aposentadoria (PPA). As estratégias para que os trabalhadores adiem sua aposentadoria não eximem o governo de sua atribuição de criar e estimular a manutenção de PPAs, fornecendo o apoio necessário à tomada dessa decisão.

\section{Referências}

Albrecht, P. A. T., \& Krawulski, E. (2011). Concurseiros e a busca por um emprego estável: reflexões sobre os motivos de ingresso no serviço público. Cadernos de Psicologia Social do Trabalho, 14(2), 211-226. 
Barnes, H., Parry, J., \& Taylor, R. (2004). Working after state pension age: qualitative research (Research Report No. 208). Recuperado de http://campaigns.dwp.gov.uk/asd/asd5/ rports2003-2004/rrep208.pdf

Bôas, B. V., \& Souza, L. (2015, 27 de setembro). Extinção de abono de permanência ameaça esvaziar órgãos públicos. Folha de São Paulo. Recuperado de http://www1.folha.uol.com. br/poder/2015/09/1687045-extincao-de-abono-ameaca-esvaziar-orgaos-publicos.shtml

Ekerdt, D. J. (2010). Frontiers of research on work and retirement. Journal of Gerontology: Social Sciences, 65B(1), 69-80. Doi:10.1093/geronb/ gbp 109

Emenda Constitucional, $n .41$, de 19 de dezembro de 2003. Modifica os arts. 37, 40, 42, 48, 96, 149 e 201 da Constituição Federal, revoga o inciso IX do $\S 3$ do art. 142 da Constituição Federal e dispositivos da Emenda Constitucional $n^{\circ} 20$, de 15 de dezembro de 1998, e dá outras providências. Recuperado de http://www.planalto. gov.br/ccivil_03/constituicao/emendas/emc/ emc41.htm

Field, A. (2009). Descobrindo a estatística usando o SPSS. Porto Alegre: Artmed.

Flynn, M. (2010). Who would delay retirement? Typologies of older workers. Personnel Review, 39(3), 308-324. Doi:10.1108/00483481011030511

França, L. H. F. P. (2009). Influências sociais nas atitudes dos 'Top' executivos em face da aposentadoria: Um estudo transcultural. Revista de Administração Contemporânea, 13(1), 17-35.

França, L. H. F. P., Menezes, G. S., Bendassolli, P. F., \& Macêdo, L. S. S. (2013). Aposentar-se ou continuar trabalhando? O que influencia esta decisão. Psicologia Ciência e Profissão, 33(3), 548-563.

França, L. H. F. P., \& Vaughan, G. (2008). Ganhos e perdas: Atitudes dos executivos brasileiros e neozelandeses frente à aposentadoria. Psicologia em Estudo, Maringá, 13(2), 207-216.
Harpaz, I. (2002). Expressing a wish to continue or stop working as related to the meaning of work. European Journal of Work and Organizational Psychology, 11(2), 177-198. Recuperado de http://www.tandfonline.com/doi/ abs/10.1080/13594320244000111

Lei $n^{\circ} 152$, de 3 de dezembro de 2015. Dispõe sobre a aposentadoria compulsória por idade, com proventos proporcionais, nos termos do inciso II do $\S 1^{\circ}$ do art. 40 da Constituição Federal. Recuperado de http://www.planalto.gov.br/ccivil_03/leis/lcp/lcp $152 . h t m$

Menezes, G. S., \& França, L. H. (2012). Preditores da Decisão da Aposentadoria por Servidores Públicos Federais. Revista Psicologia: Organizações e Trabalho, 12(3), 315-328.

Morse, N. C., \& Weiss, R. S. (1955). The function and meaning of work and the job. American Sociological Review, 20(2), 191-198.

Morin, E. M. (2001). Os sentidos do trabalho. Revista de Administração de Empresas, 41(3), 8-19.

MOW International Research Team. (1987). The meaning of working. London: Academic Press.

Organisation for Economic Cooperation and Development (2010). Avaliação da gestão de recursos humanos no governo-relatório da OCDE: Brasil: Governo Federal (portuguese version), OECD Publishing. Doi:10.1787/9789264086098-pt

Painel Estatístico de Pessoal (2018, Agosto). Ministério do Planejamento, Desenvolvimento e Gestão - MPDG. Brasil: Governo Federal. Recuperado de http://www.planejamento.gov.br/assuntos/ servidores/painel-estatistico-de-pessoal/pep/

Pengcharoen, C., \& Shultz, K. S. (2010). The influences on bridge employment decisions. International Journal of Manpower, 31(3), 322-336. Doi: 10.1108/01437721011050602

Phillipson, C. (2013). Commentary: the future of work and retirement. Human Relations, 66(1), 143-153. Doi: 10.1177/0018726712465453

Phillipson, C., \& Smith, A. (2005). Extending working life: A review of the research literature 
(Research Report No.299). Recuperado de http://statistics.dwp.gov.uk/asd/asd5/ rports2005-2006/rrep299.pdf

Pires, A. S., Ribeiro, L.V., Souza, N. V. D. O., Sá, C. M. S., Gonçalves, F. G. A., \& Santos, D. M. (2013). A permanência no mundo do trabalho de profissionais de enfermagem com possibilidade de aposentadoria. Ciência, Cuidado e Saúde, 12(2), 338-345. Doi: 10.4025/cienccuidsaude. v12i2.18298

Post, C., Schneer, J. A., Reitman, F., \& Ogilvie, D. (2013). Pathways to retirement: A career stage analysis of retirement age expectations. Human Relations, 66(1), 87-112. Doi: 10.1177/0018726712465657

Ribeiro, S. B. (2012). A força de trabalho da Fiocruz e o processo de aposentadoria. (Dissertação de Mestrado, Escola Nacional de Saúde Pública, Rio de Janeiro). Recuperado de http:// bvssp.icict.fiocruz.br/lildbi/docsonline/get. php?id=2903

Schettini, B. P., Pires, G. M. V., \& Santos, C. H. M. (2018). Previdência e reposição no serviço público civil federal do poder executivo: microssimulações. Texto para discussão $\mathrm{N}^{\circ}$ 2365. Instituto de Pesquisa Econômica Aplicada. Ministério do Planejamento, Desenvolvimento e Gestão. Brasília: Ipea. Recuperado de http://www.ipea.gov.br/cartadeconjuntura/ index.php/2018/02/27/previdencia-e-reposicao-no-servico-publico/

Shacklock, K. (2006). Extended working lives? The meaning of working to older university workers in Australia. Journal of Human Resources Development and Management, 6(2-4), 161-173. Recuperado de http://inderscience.metapress. com/index/9HQQLQ67T41LN6UG.pdf
Shacklock, K., \& Brunetto, Y. (2011). A model of older workers' intentions to continue working. Personnel Review, 40(2), 252-274. Doi:10.1108/00483481111106110

Smeaton, D., \& McKay, S. (2003). Working after state pension age: Quantitative analysis (Research Report No. 182). Recuperado de http://www. bris.ac.uk/geography/research/pfrc/themes/sse/ pfrc0305.pdf

Szinovacz, M. E., de Viney, S., \& Davey, A. (2001). Influences of family obligations and relationships on retirement variations by gender, race, and marital status. Journal of Gerontology B Psychological Sciences Social Sciences, 56(1), 20-27. Doi: 10.1093/geronb/56.1.S20

Tabachnick, B. G., \& Fidell, L. S. (2006). Using multivarieted statistics. Pearson Education.

Tuominen, E., Karisalmi, S. Takala, M., \& Kaliva, K. (2012). How do intentions affect future retirement? A case study of the finnish flexible old-age pension scheme. European Journal of Social Security, 14(2), 111-131.

Winkelmann-Gleed, A. (2012). Retirement or committed to work? Conceptualising prolonged labour market participation through organisational commitment. Employee Relations, 34(1), 80-90. Doi:10.1108/01425451211183273

Wood, A., Robertson, M., \& Wintersgill, D. (2010). A comparative review of international approaches to mandatory retirement. Norwich (UK): Department for Work and Pensions.

Zappalà, S., Depolo, M., Fraccaroli, F., Guglielmi, D., \& Sarchielli, G. (2008). Postponing job retirement? Psychosocial influences on the preference for early or late retirement. Career Development International, 13(2), 150-167. Doi:10.1108/13620430810860558

\section{Recebido: 06 de fevereiro de 2016 Aprovado: 27 de setembro de 2018}


